Presented at 2003 CEC

Anchorage, AK

September 23, 2003

\title{
A DEPOSITED MAGNETIC THERMOMETER FOR TEMPERATURES BELOW 0.1 KELVIN
}

\author{
J.G. Tuttle, T.R. Stevenson, E.R. Canavan, M.J. DiPirro, D.E. Franz, and \\ P.J. Shirron \\ NASA Goddard Space Flight Center \\ Greenbelt, Maryland, USA
}

\begin{abstract}
Magnetic thermometers are less sensitive to self-heating due to rf noise than are traditional resistive thermometers. This makes them appealing at temperatures well below 0.1 Kelvin in the operating range of many space-flight detectors. We have built and tested a magnetic thermometer which is deposited directly onto a substrate. This device, which uses the temperature dependence of iron-doped palladium's magnetic susceptibility, includes self-shielding deposited coils surrounding a sputtered palladium layer. It is read out using a SQUID to achieve high resolution. Its small size and very good heat-sinking should eventually make it useful for the temperature control of space flight detector arrays, in particular those already using SQUID readouts. The design and test results for this device are discussed.
\end{abstract}

\section{INTRODUCTION}

In the temperature range well below 0.1 Kelvin resistive thermometers are susceptible to self-heating. If shielding and filtering are inadequate, $\mathrm{rf}$ noise currents can cause falsely high indicated temperatures, and often the user is unaware of the problem. Magnetic thermometers are a good alternative for some applications. They can be driven with relatively high excitation currents and are less vulnerable to self-heating. A common type of magnetic thermometer determines a paramagnetic material's temperature-dependent magnetic susceptibility. If that material is metallic, the thermometer is generally chemically stable and has high thermal conductivity.

This type of thermometer has been studied in its conventional configuration using a cylindrical bulk palladium sample doped with iron or manganese[1,2]. We have reported our own effort to miniaturize this design for use in space-flight applications[3]. We chose iron-doped palladium for that device. The iron interacts with the palladium to produce large magnetic moments (about $10 \mu_{\mathrm{B}}$ per iron atom), and it exhibits spin-glass behavior at 


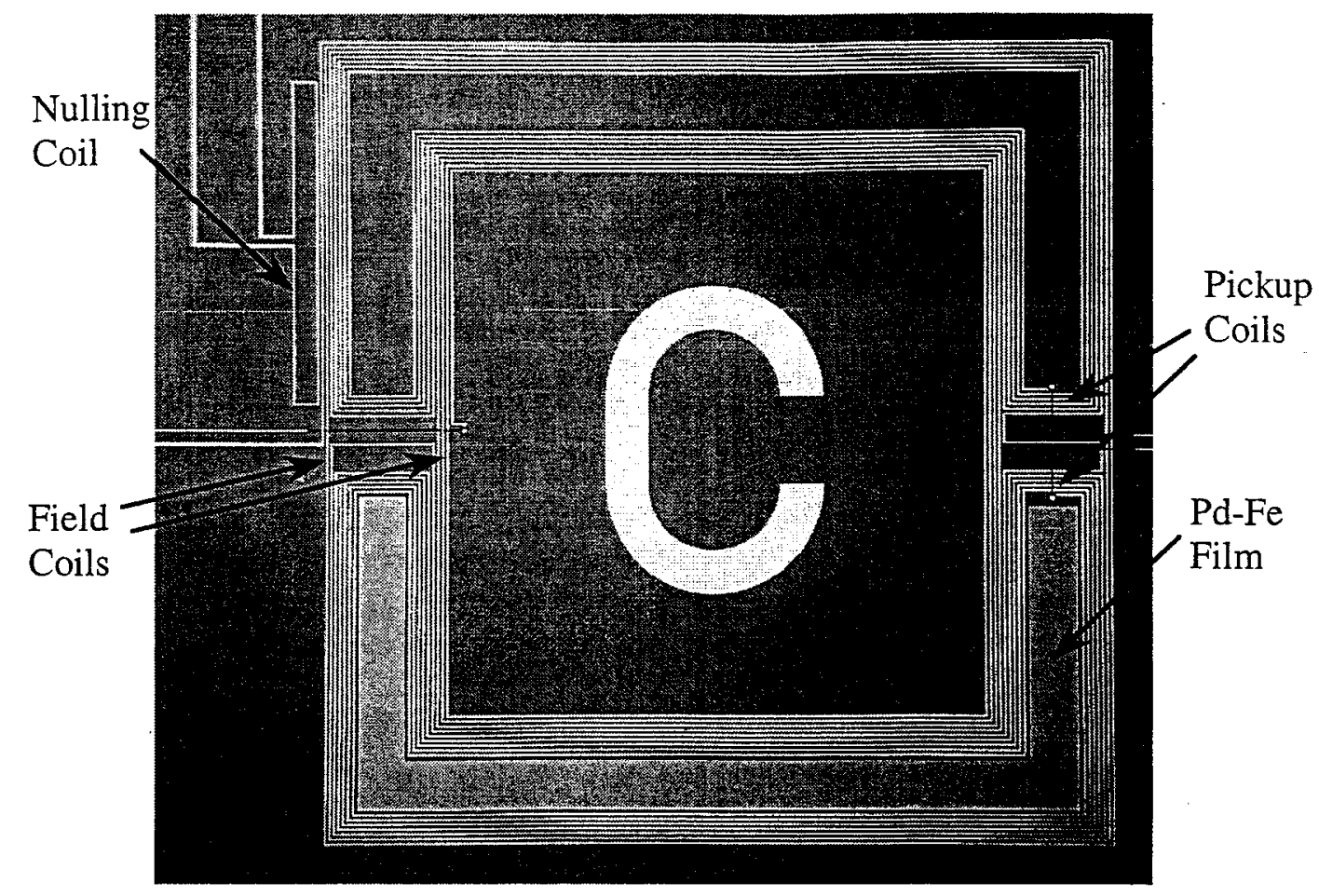

FIGURE 1. Photograph of a $1 \mathrm{~mm}$ square thermometer with a continuous Pd-Fe coating.

low temperatures[4,5]. Its magnetic susceptibility has a maximum at its freezing temperature, $\mathrm{T}_{\mathrm{F}}$, which is proportional to the iron concentration. For concentrations up to 100 atomic ppm, $\mathrm{T}_{\mathrm{F}}$ was found to be $83 \mu \mathrm{K} / \mathrm{ppm} \mathrm{Fe}[5]$. Above this temperature the susceptibility drops off, obeying a Curie-Weiss law above about $2 \cdot \mathrm{T}_{\mathrm{F}}$. We attempted to optimize the thermometer for use at $0.05 \mathrm{Kelvin}$, and it exhibited Curie-Weiss behavior above about $0.06 \mathrm{Kelvin}$. When the signal was nulled for maximum sensitivity at $\mathrm{T}=49$ $\mathrm{mK}$, we demonstrated a temperature resolution of about $40 \mathrm{nK} / \sqrt{\mathrm{Hz}}$ with an excitation current of $1 \mathrm{~mA}$ pk-pk. More recently Nelson et. al. have studied the use of sputtered films of PdMn for thermometry[6,7]. They used high concentrations of magnetic atoms $(0.7$ atomic percent) to optimize a thermometer for temperatures near $2.2 \mathrm{Kelvin}$.

NASA has planned missions for which detectors must be held at a constant operating temperature below 0.1 Kelvin. A typical temperature stability requirement for flight detectors is approximately $1 \mu \mathrm{K} / \sqrt{\mathrm{Hz}}$ for frequencies near $1 \mathrm{~Hz}$. Conventional resistive thermometers are typically used for temperature control. In addition to self-heating, these devices are difficult to heat sink to the detector substrate. In the interest of solving these problems we are developing a magnetic thermometer which is deposited directly onto a substrate. As was the case with our bulk Pd-Fe thermometer, this new device performs a mutual inductance measurement. An AC current in a field coil induces a pickup coil current, which is read out using a SQUID. The coils are well coupled (concentric), and a $\mathrm{Pd}-\mathrm{Fe}$ film is deposited inside the pickup coil. We have attempted to minimize the size, with a goal to demonstrate temperature resolution better than $1 \mu \mathrm{K} / \sqrt{\mathrm{Hz}}$.

\section{DESIGN/FABRICATION}

The actual thermometer layout can be seen in the photograph in FIGURE 1. The selfshielding field coil design, based on previous detector development work [8], consists of two concentric square coils wound in opposite directions and wired in series. The coils' 


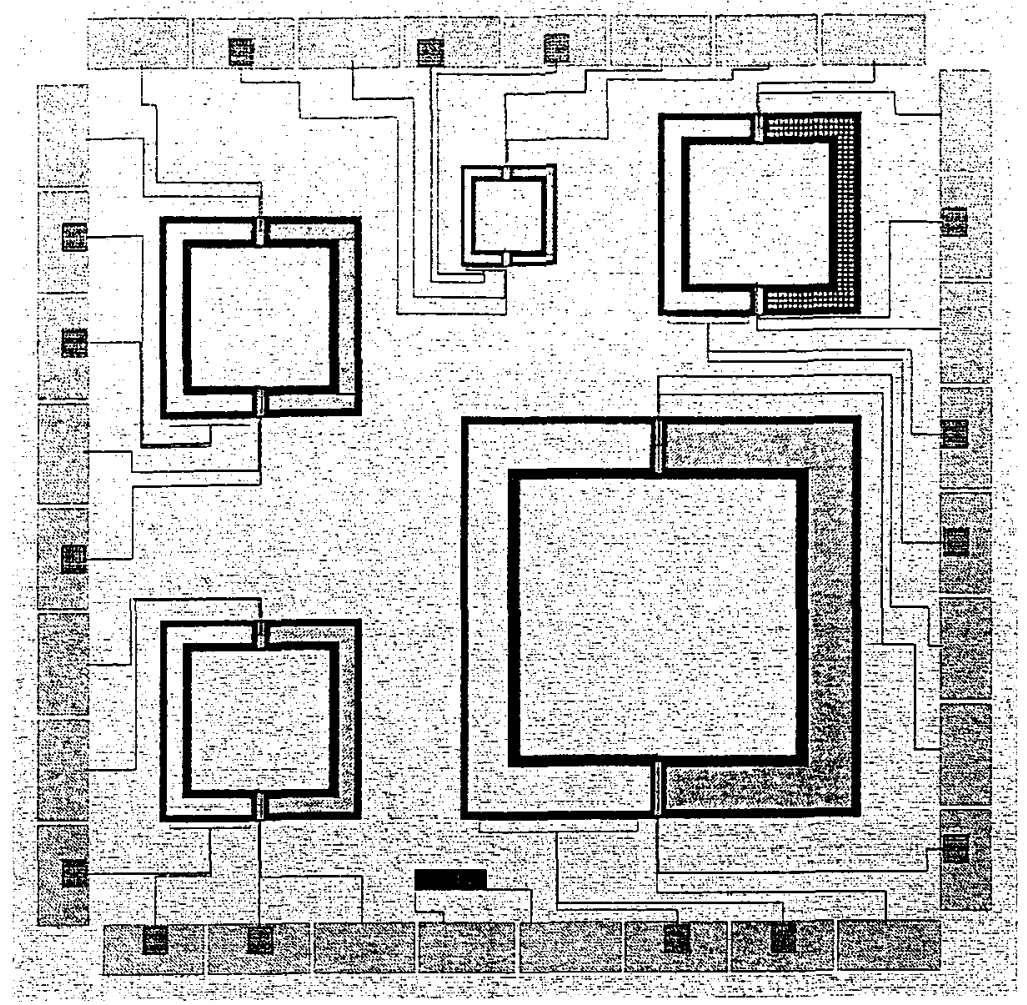

FIGURE 2. The layour of the $1 \mathrm{~cm}$ square test chip. The square thermometers range in size from $1 \mathrm{~mm}$ to $4 \mathrm{~mm}$ on a side. The top right thermometer includes the palladium grid pattern. The palladium meander line is at the chip's bottom center.

areas and numbers of windings were chosen to eliminate the production of magnetic fields far from the thermometer. The two $\mathrm{C}$-shaped pickup coils are located in the area between the field coils, where the field is maximum. The pickup coils are wound in opposing directions, and the Pd-Fe sample is sputtered onto the area inside only one of them. In this way the mutual inductance between the field and pickup coils is naturally nulled at high temperatures where the sample's magnetic susceptibility is small. The calculated magnetic field in the sample is highest at the edges and lowest in the center. For a $5 \mathrm{~mA}$ drive current, the mid-sample calculated fields are $0.58,0.58$ and 0.77 gauss for the 4,2 , and 1 $\mathrm{mm}$ coils respectively. The corresponding averaged inner and outer edge fields are 1.26 , 0.80 , and 0.94 gauss. A nulling coil, located just outside the outer field coil, allows cancellation of the signal at arbitrary temperatures for maximum sensitivity. The field and pickup coils include crossovers between different layers, while the nulling coil is entirely on one layer.

The palladium was sputtered onto the surface from a target purchased from Williams Advanced Materials of Buffalo, NY. The target was prepared with the proper doping by adding a small quantity of "Pd-Fe master" sample to a pure Pd. The target contains a total of $624 \mathrm{ppm}$ (mass) impurities, including the $200 \mathrm{ppm}$ (atomic) of iron. Except for the iron, the only measurable magnetic impurity is Nickel ( $8 \mathrm{ppm}$ by mass). The devices were shielded by a shutter during the beginning of the sputtering process in order to ensure that the iron content of the coating would be uniform. The sputtered Pd layer was $1.0 \mu \mathrm{m}$ thick.

The thermometer test chip design is shown in FIGURE 2. Each chip includes five individual thermometers ranging in size from one to four $\mathrm{mm}$ on a side. The thermometer traces lead to rectangular $\mathrm{Nb}$ pads around the chip's perimeter. The Pd-Fe in one of the $2 \mathrm{~mm}$ thermometers is deposited in a grid pattern of isolated small squares, while the other four thermometers have continuous Pd-Fe layers. The grid pattern was added to reduce the 


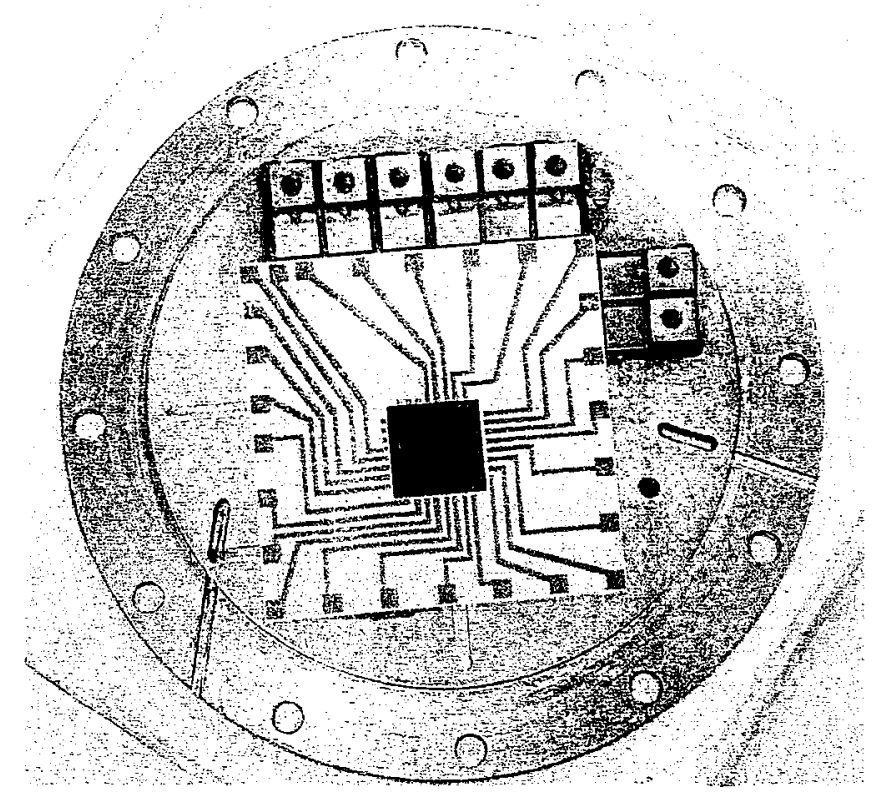

FIGURE 3. The chip is shown on its ceramic chip-holder which is mounted on the $\mathrm{Nb}$ shield base. The $\mathrm{Nb}$ terminal blocks are shown across the top and at the top-right of the chip holder.

possible pickup of signals due to Johnson noise currents in the Pd coating. A meander line of deposited Pd-Fe is included on each chip to determine its electrical resistivity. The film's RRR value turned out to be 7.2. A calculation using this value indicated that the Johnson noise pickup would be too small to resolve.

\section{TESTING}

We inspected a batch of fabricated thermometer test chips under a microscope and found several with no visible defects. On one of these the room temperature resistances across coils and interlayer connections were measured to verify that they had a high likelihood of being superconducting. The chosen chip was attached via varnish to a customized alumina chip holder, and each of the chip's pads was aluminum wire-bonded to an aluminum trace on the holder. The chip holder was in turn varnished onto a niobium plate, which formed the bottom of a niobium shield-can. This level of assembly is shown in FIGURE 3. Eight electrically-isolated niobium contact terminals were mounted on the plate using Stycast epoxy. Six of these terminals were wire-bonded to the appropriate chip holder traces so that one of the thermometers could be tested. The design allows the holder to be removed, rotated by multiples of 90 degrees, re-installed and re-bonded in order to test a different thermometer on the chip. In one of the four holder positions the remaining two terminals can be wired to the Pd-Fe meander line for its resistance measurement.

$\mathrm{The} \mathrm{Nb}$ plate was bolted to the cold stage of an adiabatic demagnetization refrigerator (ADR) which provides steady temperature control down to $30 \mathrm{mK}$. Before final closeout the $\mathrm{Nb}$ shield-can was bolted in place on the plate. Test leads from the dewar were fed to the contact terminals via right-angled holes entering through the plate's outer edge. The ends of these leads and the terminals to which they were bolted were first abraded with fine sandpaper to ensure superconducting joints. At all points external to the shield-can the leads ran through tiny stainless steel tubes coated on the outside with lead-tin solder. These 


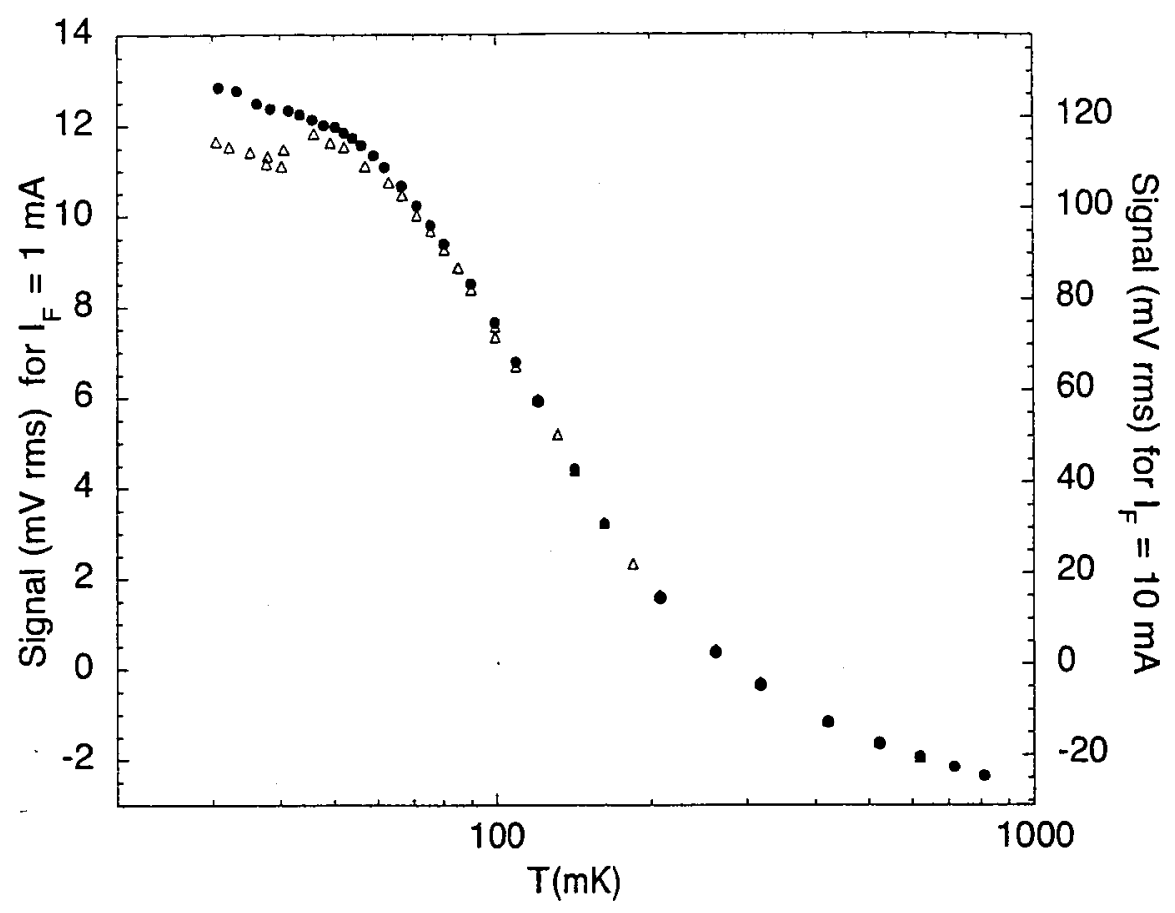

FIGURE 4. Data for the $2 \mathrm{~mm}$ thermometer. The circles and triangles were taken with $1 \mathrm{~mA}$ and $10 \mathrm{~mA}$ pk-pk field currents respectively. The y-axis scales are proportional to the field currents.

tubes provided superconducting shielding at their low-temperature ends while leaving the cold ADR stage thermally isolated.

The field and nulling coils were driven by the two channels of an HP model 3245A Universal Source. These currents could be synchronized very precisely in frequency and phase, making the nulling a fairly easy process. Both excitation currents were fed through 22 pf pi filters at the dewar main shell. The pickup coil current was read out using a Quantum Design model 5000 DC SQUID amplifier mounted on the 1.1 Kelvin stage. The SQUID controller's output signal was fed to both a spectrum analyzer and lock-in amplifier.

The first thermometer tested was a $2 \mathrm{~mm}$ device with a continuous Pd film (no grid pattern). With the thermometer's temperature held at $50 \mathrm{mK}$, the first step was to determine the excitation current's optimum frequency and amplitude. Disregarding several spikes which we assumed to be mechanical resonances in the dewar, the SQUID's output noise signal was frequency-independent up to $50 \mathrm{KHz}$. With the SQUID set for its " 1 volt per $\Phi_{0}$ " range, the noise floor was $10 \mu \mathrm{V} / \sqrt{\mathrm{Hz}}$, which was $2-3$ times the SQUID noise specification. All testing reported in this paper was done with the SQUID set for this range. By avoiding steady state spikes as well as those generated by tapping on the dewar, we identified several low-noise operating ranges and selected a favorable operating frequency of $19 \mathrm{~Hz}$.

The superconducting field coil circuit transitioned to normal when the field current was raised from 10 to $20 \mathrm{~mA}$, so the we kept the current at or below $10 \mathrm{~mA}$ during subsequent testing. In all references to field and nulling coil current in this paper, the amplitude is peak-to-peak. We studied the nulling current at $\mathrm{T}=320 \mathrm{mK}$ as a function of the field current and found it to be very linear, with $\mathrm{I}_{\text {NULL }}=-0.6 \mathrm{I}_{\text {FIELD. }}$. The signal was nulled at the arbitrarily chosen temperature of $320 \mathrm{mK}$, and thermometry data was taken at temperatures between $30 \mathrm{mK}$ and $700-900 \mathrm{mK}$ with field currents of $1 \mathrm{~mA}$ and $10 \mathrm{~mA}$.

The data are shown in FIGURE 4. On this graph the y-axis scales are proportional to the corresponding field currents, and the $10 \mathrm{~mA}$ axis and data have been shifted upward by 
$1.5 \mathrm{mV}$. With the data from the two runs effectively normalized in this way, they match quite well above about $120 \mathrm{mK}$. For lower temperatures the scaled higher-current data are systematically lower. The data clearly deviate from Curie-Weiss behavior at low temperatures. Extrapolating from other results with bulk dilute Pd-Fe samples[5], the expected spin glass freezing temperature for our film's iron concentration is about $18 \mathrm{mK}$. Thus we would expect Curie-Weiss behavior down to about $40 \mathrm{mK}$. The data above 100 $\mathrm{mK}$ fit well to the function $\mathrm{F}=\mathrm{A}+\mathrm{B} /\left(\mathrm{T}-\mathrm{T}_{\mathrm{F}}\right)$, with $\mathrm{T}_{\mathrm{F}}=18 \mathrm{mK}$.

Below about $45 \mathrm{mK}$ the $10 \mathrm{~mA}$ data were noisy and unstable, unlike the $1 \mathrm{~mA}$ points. In order to study this effect we controlled the temperature at $35 \mathrm{mK}$ and slowly stepped the field current up from $0.1 \mathrm{~mA}$ to $10 \mathrm{~mA}$, with the appropriate corresponding increases in the nulling current. Surprisingly, the data were very stable during this process and linear in the current. It is not clear why the results of a temperature sweep would differ from those of a current sweep.

The experiment was warmed up, and the chip holder was rotated so that the $4 \mathrm{~mm}$ thermometer could be tested. This configuration allowed the Pd meander line leads to be wired for the resistivity measurement. In order to verify that the aluminum wire bonds were not limiting the field current, this time we used multiple parallel wires for each bond joint.

During the initial attempt to measure the field coil circuit's critical current at $50 \mathrm{mK}$, the circuit was superconducting at $20 \mathrm{~mA}$ but went normal at $30 \mathrm{~mA}$. However, after the ADR stage cooled back down, $\mathrm{I}_{C}$ had dropped to $15 \mathrm{~mA}$, and later to about $12 \mathrm{~mA}$. Evidently driving the circuit normal caused some incremental damage. We measured the critical current as a function of temperature, taking care not to allow the chip to heat up much when it went normal. I $I_{C}$ varied little, ranging from $11.5 \mathrm{~mA}$ at $50 \mathrm{mK}$ to about $8 \mathrm{~mA}$ at 1.0 Kelvin. Next, the transition temperature of the aluminum circuit components was determined to be 1.13 Kelvin by watching the onset of Johnson noise. The weak temperature dependence of $I_{C}$ near 1 Kelvin seemed to rule out aluminum parts being the circuit's weak link.

For this run we chose an operating frequency of $88 \mathrm{~Hz}$ and kept the field current at 5 $\mathrm{mA}$. The circuit was nulled at an arbitrary temperature above $300 \mathrm{mK}$, and measurements were taken while stepping the temperature from $30 \mathrm{mK}$ up to $300 \mathrm{mK}$. The data are shown as triangles in FIGURE 5. Again, they show a higher-than-expected roll-off temperature. This time the fit to $F=A+B /\left(T-T_{F}\right)$ yielded the value: $T_{F}=-32 m K$.

We warmed up the apparatus and carefully inspected the chip under a microscope. All of the wire bond joints were intact, and the chip showed no sign of damage. A new chip was installed on the holder and wired to test its $4 \mathrm{~mm}$ thermometer. This time, after cooling to $50 \mathrm{mK}$, the field coil circuit went normal at between 4 and $5 \mathrm{~mA}$. We chose an $88 \mathrm{~Hz}$ field current of $1 \mathrm{~mA}$ and nulled the signal at $200 \mathrm{mK}$. During this run the noise floor was $13 \mu \mathrm{V} / \sqrt{\mathrm{Hz}}$, somewhat higher than during the earlier runs. The data, taken between $30 \mathrm{mK}$ and $200 \mathrm{mK}$, are shown as black circles in FIGURE 5. Again, the axes are scaled to the excitation current and positioned to align the data at $200 \mathrm{mK}$. The curves are similar in shape, but the scaled $1 \mathrm{~mA}$ data from the second chip rise approximately 15 percent more from $120 \mathrm{mK}$ down to low temperatures. Neither of these curves shows the low-temperature instability seen in the $2 \mathrm{~mm}$ coil driven with $10 \mathrm{~mA}$. 


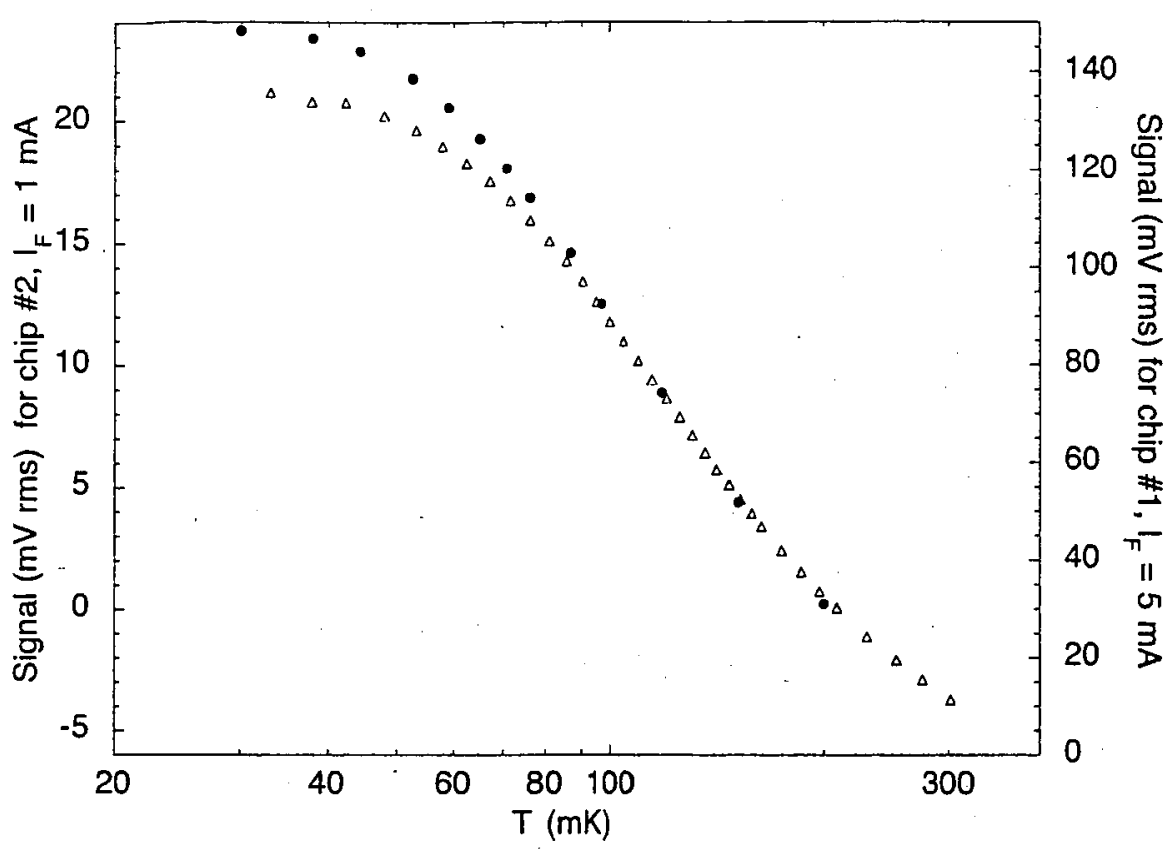

FIGURE 5. Data for the $4 \mathrm{~mm}$ thermometer. The triangles were taken with chip \#1 with a $5 \mathrm{~mA}$ field current. The circles were from chip $\# 2$ with $I_{F}=1 \mathrm{~mA}$.

Finally, we measured the nulling coil's critical current as a function of temperature from $30 \mathrm{mK}$ to 0.9 Kelvin and found it to be almost exactly $40 \mathrm{~mA}$ over this entire range. A major difference between the field and nulling coil circuits is that the latter was fabricated entirely on one layer, having no crossovers. Therefore, it seems very likely that the crossovers caused the reduced $\mathrm{I}_{\mathrm{C}}$. In future deposited thermometers the crossovers will be enlarged, and critical currents of about $40 \mathrm{~mA}$ should be attainable.

The slope of the data versus temperature gives the thermometer sensitivity in Volts/Kelvin. The maximum sensitivity occurred between 70 and $80 \mathrm{mK}$ for the four runs presented here. The noise floor $(\mathrm{V} / \sqrt{\mathrm{Hz}})$ near the operating frequency divided by the sensitivity gives the temperature resolution. TABLE 1 shows these values for the four thermometer configurations tested so far. The best demonstrated resolution was $11.1 \mu \mathrm{K} / \sqrt{\mathrm{Hz}}$ for the $4 \mathrm{~mm}$ coil tested with a field current of $5 \mathrm{~mA}$, followed closely by the $2 \mathrm{~mm}$ coil excited with twice the current. The table's last column gives the resolution expected with a field current of $40 \mathrm{~mA}$. The value for the first chip's $4 \mathrm{~mm}$ thermometer is close to the $1 \mu \mathrm{V} / \mathrm{JHz}$ goal of this project. Note that if the noise floor for the test of the second chip had been as low as that of the earlier runs, its $4 \mathrm{~mm}$ thermometer would have achieved less than $1.1 \mu \mathrm{V} / \mathrm{JHz}$ with $\mathrm{I}_{\mathrm{F}}=40 \mathrm{~mA}$.

\section{CONCLUSIONS}

Unfortunately, time constraints did not permit the testing of the $1 \mathrm{~mm}$ thermometer or the $2 \mathrm{~mm}$ sensor with the palladium grid pattern before this paper was submitted for publication. However, the data presented here have brought the work closer to it's performance goals while leaving some questions unanswered.

The field-current-dependent signal rolloff at temperatures well above $2 \cdot T_{F}$ but below $120 \mathrm{mK}$ suggests self-heating due to currents induced in the resistive palladium film. This heating would be proportional to the square of frequency, which explains the more pronounced effect at $88 \mathrm{~Hz}$. It would also be more significant at lower temperatures, as was observed. Clearly we need to do a careful study of the signal vs. temperature and field 
TABLE 1. Temperature resolution data for different test configurations

\begin{tabular}{c|c|c|c|c|c|c|c} 
Chip \# & $\begin{array}{c}\text { Coil size } \\
(\mathrm{mm})\end{array}$ & $\begin{array}{c}\text { Temp. of } \\
\text { max slope } \\
(\mathrm{mK})\end{array}$ & $\begin{array}{c}\text { Maximum } \\
\mathrm{dV} / \mathrm{dT} \\
(\mathrm{Vrms} / \mathrm{K})\end{array}$ & $\begin{array}{c}\text { Field } \\
\text { current } \\
(\mathrm{mA} \mathrm{pk}- \\
\mathrm{pk})\end{array}$ & $\begin{array}{c}\text { Noise } \\
\text { floor } \\
(\mu \mathrm{V} / \sqrt{\mathrm{Hz}})\end{array}$ & $\begin{array}{c}\text { Resolution } \\
(\mu \mathrm{K} / \sqrt{\mathrm{Hz}})\end{array}$ & $\begin{array}{c}\text { Scaled } \\
\text { resolution } \\
(\mu \mathrm{K} / \sqrt{\mathrm{Hz}})\end{array}$ \\
\hline 1 & 2 & 70 & -0.095 & 1.0 & 10 & 105 & 2.63 \\
\hline 1 & 2 & 80 & -0.88 & 10.0 & 10 & 11.4 & 2.85 \\
\hline 1 & 4 & 75 & -0.90 & 5.0 & 10 & 11.1 & 1.39 \\
\hline 2 & 4 & 70 & -0.23 & 1.0 & 13 & 57 & 1.43
\end{tabular}

current amplitude and frequency. If self-heating is confirmed, we will obviously focus on lower-frequency operation. We are also eager to test the sensor with the palladium grid pattern, as this geometry might reduce the heating effect.

Some improvements in future designs seem obvious. Enlarging the crossovers would allow higher field currents, although this might only be useful at low frequencies. The palladium film should be thicker, which would enhance the signal by increasing the number of spins. The field coils could be optimized slightly to increase the field/current ratio, and it should be possible to reduce the noise in the SQUID circuit. A combination of these changes should result in temperature resolution much better than the temperature stability required by flight projects. Any achieved margin would allow us to back off on the field current, choose a smaller thermometer size, or perhaps try a lower iron concentration in the palladium. This last option would decrease the freezing temperature and might improve the Curie-Weiss behavior in the operating range.

\section{ACKNOWLEDGEMENTS}

The authors would like to acknowledge the help provided by Carol Sappington, Evan Kunes and Brent Warner. This work was funded by Goddard Space Flight Center's Director's Discretionary Fund.

\section{REFERENCES}

1, Jutzler, M., Schroder, K., Gloos, K., and Pobell, F., Z. Phys. B, 64, pp. 115-118 (1986).

2. Klemme, B.J., Adriaans, M.J., Day, P.K., Sergatskov, T.L., Aselage, T.L., and Duncan, R.V., J Low Temp. Phys., 116, pp. 133-146 (1999).

3. Tuttle, J.G., DiPirro, M.J., Canavan, E.R., Shirron, P.J., Kunes, E., and Hait, T.P., Adv. Cryo. Eng., 47, pp. 1613-19 (2002).

4. Webb, R.A., Crabtree, G.W., and Vuillemin, J.J., Phys. Rev. Letters, 43, pp. $796-799$ (1979).

5. Peters, R.P., Buchal, C., Kubota, M., Mueller, R.M., and Pobell, F., Phys. Rev. Letters, 53, pp. 1108-1111 (1979).

6. Nelson, R.C., Sergatskov, D.A., and Duncan, R.V., J Low Temp. Phys., 126, pp. 649-654 (2002).

7. Nelson, R.C., Sergatskov, D.A., and Duncan, R.V., J Low Temp. Phys., 127, pp. 173-188 (2002).

8. Stevenson, T.R., Pellerano, F.A., Stahle, C.M., Aidala, K., and Schoelkopf, R.J., Appl. Phys. Lett., 80, pp. 3012-3014 (2002). 